\title{
A SAMELSON PRODUCT AND HOMOTOPY-ASSOCIATIVITY
}

\author{
STEPHEN J. SCHIFFMAN
}

\begin{abstract}
A certain Samelson (commutator) product is computed in the homotopy groups of a finite $\boldsymbol{H}$-space. This result is applied to the study of $H$-maps in $\pi_{3}$ of that space, and to the study of multiplications on Lie groups.
\end{abstract}

0. Introduction. Let $m: S^{3} \times S^{3} \rightarrow S^{3}$ be a multiplication on the 3-sphere $S^{3}$ and let $\alpha \in \pi_{3}\left(S^{3}\right) \cong Z$ be a generator. Recall that Arkowitz and Curjel [2] have shown that the Samelson (commutator) product $\langle\alpha, \alpha\rangle_{m} \in \pi_{6}\left(S^{3}\right) \cong$ $Z / 12$ is a generator precisely when $m$ is homotopy-associative. In this paper we shall consider a generalization (Theorem 0.1 ) of that computation and also present some applications.

We now give specific results. Unless otherwise stated $X$ will denote a finite CW $H$-space with $\pi_{3}(X) \cong Z$. Further $X$ must possess at least one homotopy-associative multiplication and satisfy a technical condition 0.4 (which holds for all known finite $H$-spaces). Included in our study are all compact, connected, simple, nonabelian Lie groups. The following is proved in $\$ 1$.

0.1 THEOREM. Let $\alpha \in \pi_{3}(X)$ be a generator. If $\mu$ is any homotopy-associative multiplication on $X$ then $\langle\alpha, \alpha\rangle_{\mu}$ generates $\pi_{6}(X)$.

As an application we consider the question raised at Neuchâtel [9, Problem 24, p. 127] as to whether or not $\alpha$ is an $H$-map, and prove in \$2:

0.2 THEOREM. (a) Given any multiplication $m$ on $S^{3}$ there exists a multiplication $\mu$ on $X$ such that $\alpha:\left(S^{3}, m\right) \rightarrow(X, \mu)$ is an $H$-map.

(b) Given any multiplication $\mu$ on $X$ there exists a multiplication $m$ on $S^{3}$ such that $\alpha:\left(S^{3}, m\right) \rightarrow(X, \mu)$ is an H-map.

In a different direction Theorem 0.1 has an application to the study of properties of multiplications on Lie groups. Recall that Mimura [6] has shown that there are $12^{15} \cdot 3^{9} \cdot 5 \cdot 7$ distinct multiplications on SU(3). We show in $\S 3$

0.3 TheOREM. At least one-third of the multiplications on $\mathrm{SU}(3)$ are not homotopy-associative.

Let us now state the condition we assume (except in §3) all finite $H$-spaces satisfy in this paper. Let $\tilde{X}$ denote the universal cover of $X$.

Received by the editors September 29, 1977.

AMS (MOS) subject classifications (1970). Primary 55D45, 55E15; Secondary 55G45.

(1) American Mathematical Society 1978 
0.4 Condition. The 5-skeleton of $\tilde{X}$ is a bouquet of spaces $S^{3}$ and $S^{3} \cup_{\eta} e^{5}$.

This condition is conjectured true in general by Harper (cf. [4, Proposition 3.4]) and we assume it to be able to make use in $\S 1$ of a result from [4].

For the remainder of the introduction we give notation. We work in the homotopy category of path-connected based spaces of the homotopy type of $\mathrm{CW}$-complexes and do not distinguish between maps and homotopy classes of maps. The notation $Z / n$ is used for the integers $\bmod n$ and $G_{p}$ and $Y_{p}$ for the localizations at a prime $p$ of, respectively, an abelian group $G$ and a 1connected space $Y$. For basic facts about localizations see [11], and for basic $H$-space definitions see [8].

\section{Proof of Theorem 0.1.}

1.1 Outline of proof. Let $X$ denote a finite $H$-space satisfying Condition 0.4 with $\pi_{3}(X) \cong Z$ generated by $\alpha$, and let $\mu$ be any homotopy-associative multiplication on $X$. For the purpose of computing $\langle\alpha, \alpha\rangle_{\mu} \in \pi_{6}(X)$ we may also assume that $X$ is 1 -connected. For if not, the universal cover $\tilde{X}$ of $X$ is again a finite $H$-space $(\tilde{X}, \tilde{\mu})$ with $\tilde{\mu}$ again homotopy-associative.

Now let $P$ denote Stasheff's projective plane [10] of $(X, \mu)$ and let, ambiguously, $\alpha \in \pi_{4}(P)$ correspond to $\alpha \in \pi_{3}(X)$. (Of course $\pi_{i+1}(P) \cong$ $\pi_{i}(X)$ for $i<10$.) Then since $\mu$ is homotopy-associative it is well known that the Samelson product $\langle\alpha, \alpha\rangle_{\mu}$ corresponds to the Whitehead product $[\alpha, \alpha] \in$ $\pi_{7}(P)$. We compute this Whitehead product using a technique of Arkowitz [1].

In this direction first note that, if $Y^{[n]}$ denotes the $n$-Postnikov section of a space $Y$, the 6-section map $P \rightarrow P^{[6]}$ is an isomorphism on homotopy groups in dimensions less than seven. Since $\pi_{2}(X)=0$ by [3], the space $P^{[6]}$ has nonvanishing homotopy groups only in (at most) dimensions four, five and six. For these dimensional reasons, then, $P^{[6]}$ is a loop-space. Thus the method of Arkowitz can be applied to the pair $\left(P^{[6]}, P\right)$ to give that $[\alpha, \alpha]=$ $\theta(\beta * \beta)$ where $\beta$ is a generator of $H_{4}\left(P^{[6]}\right) \cong Z$, "**" denotes Pontrjagin multiplication in $H_{*}\left(P^{[6]}\right)$, and the homomorphism $\theta$ is given as $l_{*}: H_{8}\left(P^{[6]}\right)$ $\rightarrow H_{8}\left(\pi_{7}(P), 8\right)$ where $l \in H^{8}\left(P^{[6]} ; \pi_{7}(P)\right)$ is the appropriate $k$-invariant of $P$.

We now begin detailed calculation of $l_{*}(\beta * \beta)$ by determining some low dimensional homotopy and homology groups of a 1-connected finite $H$-space $X$. By way of notation let $X^{(n)}$ denote the $n$-skeleton of $X$.

1.2 Some homotopy and homology of $X$. Set $K=S^{3} \cup_{\eta} e^{5}$ where $\eta$ is the essential map $\eta: S^{4} \rightarrow S^{3}$. Choose a generator $\gamma: S^{5} \rightarrow K$ of the infinite cyclic group $\pi_{5}(K)$ and set $L=K \cup_{\gamma} e^{6}$. By Condition 0.4 and since $\pi_{3}(X) \cong Z$, the 5-skeleton $X^{(5)}$ of $X$ is either $S^{3}$ or $K$ depending on whether $\pi_{4}(X)$ is $Z / 2$ or 0 . We next consider the possibilities for $X^{(6)}$. Since $H_{3}(X)$ is free of rank one the group $H_{6}(X)$ must be a torsion group, but by [4, Lemma 2.4] the group $H_{6}(X)$ is always torsion free, hence $H_{6}(X)=0$. Thus if $H_{5}(X)$ has no torsion then $X^{(6)}=X^{(5)}$. In case of torsion then $H_{5}(X) \cong Z / 2$ and $\pi_{5}(X)=$ 
0 again by [4, Theorem 1.2]. Therefore $X^{(5)}=K$ and $X^{(6)}=L$. We complete this analysis by noting that the inclusion $X^{(6)} \subset X$ induces an isomorphism on homotopy groups in dimensions less than 6 and an epimorphism in dimension 6. Thus if $X^{(6)}=S^{3}$ then $\pi_{6}(X)$ is a quotient of $Z / 12$, if $X^{(6)}=K$ it is a quotient of $Z / 6$, and if $X^{(6)}=L$ it is a quotient of $Z / 3$.

Noticing that at any rate $\pi_{6}(X)$ is a quotient of $Z / 12$ we now divide our calculation of $[\alpha, \alpha]$ into two parts-in 1.3 we show $[\alpha, \alpha]$ generates the 3-torsion, and in 1.4 the 2-torsion, of $\pi_{7}(P)$. To this end we use the technique of localization. Specifically, equating $\alpha \in \pi_{4}(P)$ with its localized image in $\pi_{4}\left(P_{p}\right)$ we have that $[\alpha, \alpha]$ generates the $p$-torsion in $\pi_{7}(P)$ if and only if $[\alpha, \alpha]$ generates $\pi_{7}\left(P_{p}\right)$.

1.3 LEMMA. $[\alpha, \alpha]$ generates the 3-torsion of $\pi_{7}(P)$.

Proof. In this proof all spaces have been localized at the prime 3 but if $Y$ denotes any space we continue to write $Y$ for $Y_{3}$. Assume, to avoid trivialities, that $Z / 3 \subset \pi_{7}(P)$. Then by 1.2 we have that if $X^{(6)}$ is $S^{3}$ or $L$ then $\pi_{4}(P) \cong Z_{3}, \pi_{7}(P) \cong Z / 3$, and $\pi_{i}(P)=0$ for all other $i<7$. Or if $X^{(6)}$ is $K$ then $\pi_{4}(P) \cong \pi_{6}(P) \cong Z_{3}, \pi_{7}(P) \cong Z / 3$, and $\pi_{i}(P)=0$ for all other $i<7$. Thus in the former case $P^{[6]}=K\left(Z_{3}, 4\right)$ and in the latter case, since the only possible $k$-invariant lies in a zero group, $P^{[6]}=K\left(Z_{3}, 4\right) \times K\left(Z_{3}, 6\right)$. In either case, if $\beta$ is a generator of $H_{4}\left(P^{[6]}\right) \cong Z_{3}$-that is, $3 \nmid \beta$-then $\beta * \beta=$ $2 \gamma_{2}(\beta)$ where $\gamma_{2}: H_{4}\left(P^{[6]}\right) \cong H_{4}\left(Z_{3}, 4\right) \rightarrow H_{8}\left(Z_{3}, 4\right) \cong H_{8}\left(P^{[6]}\right)$ is the second divided power in the ring $H_{*}\left(Z_{3}, 4\right)$.

Now let $y \in H^{4}\left(P^{[6]} ; Z / 3\right) \cong Z / 3$ be the basic class. We show in $\$ 4$ that the $k$-invariant $l \in H^{8}\left(P^{[6]} ; Z / 3\right)$ is always given by $l=a \mathcal{P}^{1} y+b y^{2}$ where $3 \nmid a$ and $3 \nmid b$. Then by 1.1

$$
\begin{aligned}
{[\alpha, \alpha] } & =l_{*}(\beta * \beta)=\left(a \mathcal{P l}^{1} y+b y^{2}\right)_{*}(\beta * \beta) \\
& =\left(a \mathscr{P}^{1} y\right)_{*}(\beta * \beta)+\left(b y^{2}\right)_{*}(\beta * \beta)
\end{aligned}
$$

(this since if $f$ and $g$ are maps $A \rightarrow B$ where $B$ is an $n$-connected $H$-space, then $(f+g)_{*}=f_{*}+g_{*}$ on homology in dimensions less than $\left.2 n+2\right)$

$$
=\left(a^{\mathcal{P}} y\right)_{*}(\beta) *\left(a \mathcal{P}^{1} y\right)_{*}(\beta)+\left(b y^{2}\right)_{*}(\beta * \beta)
$$

(this since $\mathcal{P P}^{\mathrm{l} y}$ is primitive)

$$
=\left(b y^{2}\right)_{*}(\beta * \beta)
$$

(this since $\left(\mathcal{P}^{1} y\right)_{*}(\beta) \in H_{4}(Z / 3,8)=0$ )

$$
=2 b y_{*}^{2}\left(\gamma_{2}(\beta)\right) \text { which is a generator of } \pi_{7}(P)
$$

since $y_{*}^{2}\left(\gamma_{2}(\beta)\right)$ is a generator of $H_{8}(Z / 3,8) \cong \pi_{7}(P)$. Q.E.D.

1.4 LEMMA. $[\alpha, \alpha]$ generates the 2-torsion of $\pi_{7}(P)$.

Proof. In this proof all spaces have been localized at the prime 2 but if $Y$ denotes any space we continue to write $Y$ for $Y_{2}$. Again to avoid trivialities 
assume $Z / 2 \subset \pi_{7}(P)$. By 1.2 we need only consider the two cases $X^{(6)}=S^{3}$ or $X^{(6)}=K$.

It may be determined in the first case that $H_{8}\left(P^{[6]}\right) \cong Z_{2} \oplus Z / 4$ and in the second case that $H_{8}\left(P^{[6]}\right) \cong Z_{2} \oplus Z / 2$. Furthermore in both cases if $\beta$ is a generator of $H_{4}\left(P^{[6]}\right) \cong Z_{2}$-that is, $2 \nmid \beta$-then $\beta * \beta=2 \gamma_{2}(\beta)+u \in H_{8}\left(P^{[6]}\right)$ where $\gamma_{2}: H_{4}\left(P^{[6]}\right) \cong H_{4}\left(Z_{2}, 4\right) \rightarrow H_{8}\left(Z_{2}, 4\right) \cong Z_{2} \subset H_{8}\left(P^{[6]}\right)$ is the second divided power in the ring $H_{*}\left(Z_{2}, 4\right)$ and where $u$ is a generator of the appropriate $Z / 4$ or $Z / 2$ summand in $H_{8}\left(P^{[6]}\right)$. We omit calculation of these facts.

We have left to determine the $k$-invariant $l \in H^{8}\left(P^{[6]} ; \pi_{7}(P)\right)$. Suppose $\pi_{7}(P) \cong Z / 2$; we consider the remaining case $\pi_{7}(P) \cong Z / 4$ later. Then $H^{8}\left(P^{[6]} ; Z / 2\right)$ is isomorphic to $\operatorname{Hom}\left(H_{8}\left(P^{[6]}\right), Z / 2\right) \cong Z / 2 \oplus Z / 2$ with generators $z$ and $x$ such that $z_{*}\left(\gamma_{2}(\beta)\right)=w, z_{*}(u)=0$ and $x_{*}\left(\gamma_{2}(\beta)\right)=0$, $x_{*}(u)=w$ where $w$ generates $Z / 2 \cong H_{8}(Z / 2,8)$. It follows that $z$ is the element $y^{2}$ where $y \in H^{4}\left(P^{[6]} ; Z / 2\right) \cong Z / 2$ is the basic class.

Thus the $k$-invariant $l$ is either $0, y^{2}, y^{2}+x$, or $x$. An easy calculation rules out 0 and $y^{2}$ as choices for $l$ and in each of the two remaining cases we may now compute that $[\alpha, \alpha]=l_{*}(\beta * \beta)=x_{*}\left(2 \gamma_{2}(\beta)+u\right)=w$ which generates $H_{8}(Z / 2,8) \cong \pi_{7}(P)$.

Finally we have left to consider the case $\pi_{7}(P) \cong Z / 4$. We can show that $\langle\alpha, \alpha\rangle_{\mu}$ generates the 2-torsion of $\pi_{6}(X)$ directly in this case by noting that here the 6-Postnikov section $X^{[6]}=\left(S^{3}\right)^{[6]}$ (recall all spaces are 2-local). For then it is known [2, Lemma 4(c)] that, with respect to any multiplication $m$ on $S^{3},\langle\gamma, \gamma\rangle_{m}$ generates the 2-torsion of $\pi_{6}\left(S^{3}\right)$ for any generator $\gamma \in \pi_{3}\left(S^{3}\right)$. This concludes the proof of 1.4 and with it the proof of Theorem 0.1 .

2. Proof of Theorem 0.2. Recall our Condition 0.4 on $X$, and that $X$ possesses at least one homotopy-associative multiplication, and that $\pi_{3}(X) \cong$ $Z$ with generator $\alpha$. We shall need the following corollary of Theorem 0.1 : Let $S=S^{3}$ throughout this section.

2.1 LemMA. The homomorphism $\alpha \wedge \alpha^{*}:[X \wedge X, X] \rightarrow[S \wedge S, X]$ is an epimorphism.

Proof. If $1: X \rightarrow X$ is the identity map then $\alpha \wedge \alpha^{*}\langle 1,1\rangle_{\mu}=\langle\alpha, \alpha\rangle_{\mu}$, which, by 0.2 , generates $[S \wedge S, X] \cong \pi_{6}(X)$ for any homotopy-associative multiplication $\mu$. Q.E.D.

2.2 Proof of TheOrem 0.2 (a). Fix any homotopy-associative multiplication $\mu_{0}$ on $X$. Then any multiplication $\mu$ on $X$ is given by $\mu=\mu_{0} \cdot q^{*}(\eta)$ for some map $\eta: X \wedge X \rightarrow X$ and where $q: X \times X \rightarrow X \wedge X$ denotes projection and where the multiplication "." is taken with respect to $\mu_{0}$.

We must find an $\eta$ such that

$$
\begin{aligned}
& \alpha \circ m=\mu_{0} \cdot q^{*}(\eta) \circ \alpha \times \alpha \Leftrightarrow \\
& \alpha \circ m=\left(\mu_{0} \circ \alpha \times \alpha\right) \cdot\left(q^{*}(\eta) \circ \alpha \times \alpha\right) .
\end{aligned}
$$

But the map $\left(\mu_{0} \circ(\alpha \times \alpha)\right)^{-1} \cdot(\alpha \circ m): S \times S \rightarrow X$ is null-homotopic when 
restricted to $S \vee S$. Hence there is a map $\theta: S \wedge S \rightarrow X$ such that $p^{*}(\theta)=$ $\left(\mu_{0} \circ \alpha \times \alpha\right)^{-1} \cdot(\alpha \circ m)$ where $p: S \times S \rightarrow S \wedge S$ denotes projection.

Thus it suffices to find $\eta$ such that $p^{*}(\theta)=q^{*}(\eta) \circ \alpha \times \alpha$, which is equivalent to requiring $p^{*}(\theta)=p^{*}\left(\alpha \wedge \alpha^{*}(\eta)\right)$. Finally since $\alpha \wedge \alpha^{*}$ is epimorphic by Lemma 2.1, there exists an $\eta \in[X \wedge X, X]$ so that $\theta=\alpha \wedge$ $\alpha^{*}(\eta)$. Q.E.D.

The next lemma follows easily from the discussion in 1.2 and is given without proof.

2.4 LEMMA. The homomorphism $\alpha_{*}: \pi_{6}(S) \rightarrow \pi_{6}(X)$ is an epimorphism.

2.5 Proof of Theorem 0.2 (b). Fix any multiplication $m_{0}$ on $S$. Then by Theorem 0.2 (a) there exists a multiplication $\mu_{0}$ on $X$ such that $\alpha:\left(S, m_{0}\right) \rightarrow$ $\left(X, \mu_{0}\right)$ is an $H$-map. Now write the given multiplication $\mu$ as $\mu=\mu_{0} \cdot q^{*}(\eta)$ for some choice of $\eta: X \wedge X \rightarrow X$.

Our task is to find a map $\sigma: S \wedge S \rightarrow S$ and thus a multiplication $m=m_{0} \cdot p^{*}(\sigma)$ on $S$ so that

$$
\alpha \circ m_{0} \cdot p^{*}(\sigma)=\mu_{0} \cdot q^{*}(\eta) \circ \alpha \times \alpha
$$

(the multiplication "." in $m_{0} \cdot p^{*}(\sigma)$ is taken with respect to $m_{0}$ ).

Because we chose $\mu_{0}$ to make $\alpha:\left(S, m_{0}\right) \rightarrow\left(X, \mu_{0}\right)$ an $H$-map, the equation (2.6) is equivalent to

$$
\left(\alpha \circ m_{0}\right) \cdot\left(\alpha \circ p^{*}(\sigma)\right)=\left(\mu_{0} \circ \alpha \times \alpha\right) \cdot\left(q^{*}(\eta) \circ \alpha \times \alpha\right) .
$$

Now by [5] for any $H$-space $\left(X, \mu_{0}\right)$ the loop $[S \times S, X]$ is in fact a group. Therefore, again since $\alpha$ is an $H$-map, the equation (2.7) is equivalent to $\alpha \circ p^{*}(\sigma)=q^{*}(\eta) \circ \alpha \times \alpha$ which is equivalent to $p^{*}\left(\alpha_{*}(\sigma)\right)=p^{*}\left(\alpha \wedge \alpha^{*}(\eta)\right)$. Finally since $\alpha_{*}: \pi_{6}(S) \rightarrow \pi_{6}(X)$ is epimorphic by Lemma 2.4 , there exists a $\sigma \in \pi_{6}(S)$ so that $\alpha_{*}(\sigma)=\alpha \wedge \alpha^{*}(\eta)$. Q.E.D.

3. Homotopy-associativity in SU(3). In this section we shall prove Theorem 0.3. We begin with a simple lemma on Samelson products whose proof we omit. Consider an arbitrary homotopy-abelian $H$-space $\left(Y, m_{0}\right)$. For the purpose of $\$ 3$ only we do not place any restrictions on the space $Y$; in particular $Y$ is not required to be a finite complex. For any $\eta \in[Y \wedge Y, Y]$ denote the multiplication $m_{\eta}$ on $Y$ by $m_{\eta}=q^{*}(\eta)+m_{0}$ where " + " is multiplication in $[Y \times Y, Y]$ with respect to $m_{0}$ written additively, and $q$ : $Y \times Y \rightarrow Y \wedge Y$ denotes projection.

3.1 Lemma. Let $\alpha$ be an arbitrary element in $\pi_{3}(Y)$. Then the Samelson product $\langle\alpha, \alpha\rangle_{m_{7}}=0$ if and only if $2(\alpha \wedge \alpha)^{*}(\eta)=0$ in $\pi_{6}(Y)$.

3.2 Proof of Theorem 0.3. Let $Y$ denote the 3-localization of the Lie group $\mathrm{SU}(3) ; Y$ is easily seen to possess a homotopy-abelian multiplication $m_{0}$. Let $\alpha \in \pi_{3}(Y) \cong Z_{3}$ be such that $3 \nmid \alpha$ and consider the function $(\alpha \wedge$ $\alpha)^{*}:[Y \wedge Y, Y] \rightarrow \pi_{6}(Y) \cong Z / 3$. We consider $(\alpha \wedge \alpha)^{*}$ a homomorphism of groups with respect to the 3-localization of the standard Lie group multiplication on $\mathrm{SU}(3)$. 
Now suppose $\eta \in \operatorname{ker}(\alpha \wedge \alpha)^{*}$. Then on one hand, by Lemma 3.1, $\langle\alpha, \alpha\rangle_{m}=0$. But on the other hand it follows from Theorem 0.1 that if $m_{\eta}=q^{*}(\eta)+m_{0}$ is homotopy-associative then $\langle\alpha, \alpha\rangle_{m_{\eta}} \neq 0$. Therefore if $\eta \in \operatorname{ker}(\alpha \wedge \alpha)^{*}$ then $m_{\eta}$ is not homotopy-associative. By Mimura [6] it follows that $|[Y \wedge Y, Y]|=3^{9}$ so that $\left|\operatorname{ker}(\alpha \wedge \alpha)^{*}\right|=3^{8}$ by Lemma 2.1 . Theorem 0.3 is now a consequence of the fact that a multiplication $\mu$ on a space $X$ is homotopy-associative only if it is again homotopy-associative when localized at any prime $p$.

4. The $k$-invariant $l$. We employ the notation and conventions of $\$ 1.3$ (in particular all spaces are 3-local). In this section we compute the $k$-invariant $l \in H^{8}\left(P^{[6]} ; \pi_{7}(P)\right)$ to be $l=a^{P^{1} y}+b y^{2}$ where $y$ is a generator of $H^{4}\left(P^{[6]} ; \pi_{7}(P)\right)$ and $3 \nmid a$ and $3 \nmid b$.

The $k$-invariant is obtained (see for example [7, Chapter 13]) by considering the 6-Postnikov section map $g: P \rightarrow P^{[6]}$ and letting $F$ denote the fibre of $g$ to get the fibration $F \rightarrow P \rightarrow P^{6}$. Then $l$ is given from the Serre exact sequence of this fibration

$$
H^{7}\left(F ; \pi_{7}(P)\right) \stackrel{\tau}{\rightarrow} H^{8}\left(P^{[6]} ; \pi_{7}(P)\right) \stackrel{g^{*}}{\rightarrow} H^{8}\left(P ; \pi_{7}(P)\right)
$$

by $l=\tau(i)$ where $\tau$ is transgression and $i$ is the basic class in $H^{7}\left(F ; \pi_{7}(P)\right)$.

From the possibilities for $P$ and $P^{[6]}$ determined in $\S 1$ we have that $\pi_{7}(P) \cong Z / 3$ and $H^{8}\left(P^{[6]} ; Z / 3\right) \cong Z / 3 \oplus Z / 3$ with generators $\mathcal{P P}^{1} y$ and $y^{2}$ where $y$ is a generator of $H^{4}\left(P^{[6]} ; Z / 3\right) \cong Z / 3$. Then $H^{7}(F ; Z / 3) \cong Z / 3$ and the computation of $l$ will follow easily if we can show that $\mathcal{P}^{1} g^{*}(y)$ and $g^{*}(y)^{2}$ are linearly dependent in $H^{8}(P ; Z / 3)$.

To this end first note that since $\mu$ is homotopy-associative the third projective space $X P(3)$ of $(X, \mu)$ exists. From now on write $H^{*}()$ for $H^{*}(; Z / 3)$ and write $x=g^{*}(y) \in H^{4}(P) \cong H^{4}(X P(3))$. It is known that $0 \neq x^{3} \in H^{12}(X P(3))$. Then since $\mathscr{P}^{2} x=x^{3} \neq 0$ and since $\mathscr{P}^{1} \mathscr{P}^{1}=-\mathscr{P}^{2}$ we have that $0 \neq \mathscr{P}^{1} x \in H^{8}(P)$.

Now consider the $H^{*}$ ( ) spectral sequence of the fibration $X \rightarrow Q \rightarrow P$ where $Q$ denotes the 3 -fold join of $X$. Thereby obtain the exact sequence $E_{4}^{4,3} \rightarrow E_{4}^{8,0} \rightarrow E_{5}^{8,0} \rightarrow 0$ which can be identified with the exact sequence

$$
H^{4}(P) \otimes H^{3}(X) \stackrel{\lambda}{\rightarrow} H^{8}(P) \stackrel{\rho}{\rightarrow} E_{\infty}^{8,0} \rightarrow 0 .
$$

Here $\lambda(d \otimes e)=d \cup \tau(e)$ for any $d \in H^{4}(P)$ and $e \in H^{3}(X)$, and $\rho(w)$ is defined to be the class of $w \in H^{8}(P) \cong E_{2}^{8,0}$ in the quotient group $E_{\infty}^{8,0}$.

Let $z$ be a generator of $H^{3}(X)$ such that $\tau(z)=x=g^{*}(y)$. Then by naturality $\rho\left(\mathscr{P}^{1} x\right)=\rho\left(\mathscr{P}^{1} \tau(z)\right)=\rho\left(\tau\left(\mathcal{P}^{1} z\right)\right)$. But by [4, Theorem 1.4], the operation $\mathscr{\rho}^{1}$ is zero on $H^{3}(X)$ since $\pi_{6}(X) \cong Z / 3$. So $\left.\rho^{\left(\mathcal{P}^{1}\right.} x\right)=\rho(\tau(0))=0$. Therefore by (4.2), $0 \neq \mathscr{P}^{1} x=\lambda(v)$ for some $v \in H^{4}(P) \otimes H^{3}(X) \simeq Z / 3$. Finally by writing $v$ as $c(x \otimes z)$ for some $c$ where $3 \nmid c$ we conclude that $\mathscr{P}^{1} x=c x^{2}$.

We finish the computation of $l$. Choose $c$ as above and obtain $g^{*}\left(\mathcal{P}^{1} y-\right.$ $\left.c y^{2}\right)=\mathscr{P}^{1} x-c x^{2}=0$. By the exactness of (4.1) there is a $u \in H^{i}(F ; Z / 3) \cong$ 
$Z / 3$ so that $\tau(u)=\mathscr{P}^{1} y-c y^{2}$. But the basic class $i=k u$ for some $k$ where $3 \nmid k$. Therefore $l=\tau(i)=k^{\mathcal{P}} y-k c y^{2}$. We are done by setting $a=k$ and $b=-k c$. Q.E.D.

\section{REFERENCES}

1. M. Arkowitz, Whitehead products as images of Pontrjagin products, Trans. Amer. Math. Soc. 158 (1971), 453-463.

2. M. Arkowitz and C. R. Curjel, Some properties of the exotic multiplications on the three-sphere, Quart. J. Math. Oxford Ser. (2) 20 (1969), 171-176.

3. W. Browder, Torsion in H-spaces.I, Ann. of Math. 74 (1961), $24-51$.

4. J. Harper, Homotopy groups of H-spaces, Comment. Math. Helv. 47 (1972), 311-331.

5. P. Hilton, Note on H-spaces and nilpotency, Bull. Acad. Polon. Sci. 11 (1963), 505-509.

6. M. Mimura, On the number of multiplications on $\mathrm{SU}(3)$ and $\mathrm{Sp}(2)$, Trans. Amer. Math. Soc. 146 (1969), 473-492.

7. R. Mosher and M. Tangora, Cohomology operations and applications in homotopy theory, Harper \& Row, New York, 1968.

8. J. Stasheff, H-spaces from a homotopy point of view, Lecture Notes in Math., vol. 161, Springer-Verlag, Berlin and New York, 1970.

9. __ H-space problems, H-spaces, Neuchâtel, Août 1970, Lecture Notes in Math., vol. 196, Springer-Verlag, Berlin and New York, 1971.

10. On homotopy abelian H-spaces, Proc. Cambridge Philos. Soc. 57 (1961), 734-745.

11. D. Sullivan, Genetics of homotopy theory and the Adams conjecture, Ann. of Math. (2) 100 (1974), 1-79.

Department of Mathematics, Colorado College, Colorado Springs, Colorado 80903 